\title{
The yellow mealworm (Tenebrio molitor) genome: a resource for the emerging insects as food and feed industry
}

\author{
T. Eriksson ${ }^{1,2}$, A.A. Andere ${ }^{1}$, H. Kelstrup ${ }^{2}$, V.J. Emery ${ }^{2}$ and C.J. Picard ${ }^{1^{*}}$ \\ ${ }^{1}$ Department of Biology, Indiana University Purdue University Indianapolis, 723 W. Michigan Street, Indianapolis, IN \\ 46202, USA; ${ }^{2}$ Beta Hatch Inc., Cashmere, Washington, 200 Titchenal Road, Cashmere, WA 98815, USA; cpicard@iupui.edu
}

Received: 25 November 2019 / Accepted: 05 May 2020

(C) 2020 Wageningen Academic Publishers

OPEN ACCESS CC) (i)(-) RESEARCH ARTICLE

\begin{abstract}
The yellow mealworm, the larval stage of the darkling beetle Tenebrio molitor, shows great promise as an alternative source of animal protein. Herein we present the $312 \mathrm{Mb}$ draft genome assembled using 10x Genomics linked-read technology to inform research efforts and to provide resources to optimise yellow mealworm for mass production and consumption. The genome with a contig N50 of 39,478 bp contains $89 \%$ of conserved arthropod genes among the $>20,000$ genes assembled (complete and partial genes). This draft assembly represents a valuable resource to understanding T. molitor biology as a means of producing alternative, sustainable protein for the growing population and in the face of changing climates.
\end{abstract}

Keywords: darkling beetle, genomics, sequencing, assembly, linked-reads

\section{Introduction}

Global food demands are rising at an alarming rate, and the lack of reliable, sustainable protein sources are an emerging food security issue across the world (FAO, 2017). Thus, alternatives to traditional protein production are a necessity. One such alternative is the use of mass-produced insects for food and animal feed. While great strides are being made in mass rearing insects for consumption, many challenges associated with commercial production remain, including the optimisation of desirable life history traits such as growth rate and fecundity. Historically a time-consuming endeavour through trial-and-error selective breeding, trait optimisation can now be significantly facilitated by studying and manipulating an organism's most basic feature: its genome.

Beetles (Coleoptera) make up nearly a quarter of Earth's biodiversity and possess complex evolutionary histories, as well as a remarkable richness in morphology and lifestyle (Barton et al., 2011; Beutel and Leschen, 2014; Mckenna et al., 2015; Zhang et al., 2011). Relatively few beetle genomes have been made public (McKenna, 2018), with the first published genome belonging to the stored grain pest, the red flour beetle Tribolium castaneum (Coleoptera:
Tenebrionidae) (McKenna, 2018; Richards et al., 2008). Similarly, the darkling beetle Tenebrio molitor (Coleoptera: Tenebrionidae) and its associated larval form, the yellow mealworm, is a relative of T. castaneum and also considered a stored grain pest. However, the yellow mealworm possesses many qualities that can benefit a wide range of agricultural applications. High protein content and relative ease in rearing have made yellow mealworms a popular staple for the exotic pet food market for over 60 years. More recently, these larvae have been shown to be a suitable dietary supplement in the aquaculture and poultry industries (Choi et al., 2018; Henry et al., 2018; Veldkamp and Bosch, 2015). In addition, mealworm colonies are capable of breaking down organic materials, including mycotoxins (Van Broekhoven et al., 2017), and excreting large quantities of nitrogen-rich frass, making them ideal producers of organic fertiliser (Poveda $e t$ al., 2019). Lastly, there is evidence that farming mealworms is more sustainable than traditional livestock (Oonincx and De Boer, 2012).

T. molitor belongs to the family of detritivorous beetles Tenebrionidae, the seventh-most speciose taxon in Coleoptera comprising about 20,000 species in 2,300 genera worldwide (Zhang et al., 2011). Although it remains unclear how distantly related they are, some estimates have placed 
the respective most-recent-common ancestors of the tribes Tenebrionii and Tribolini circa 120 and 100 million years ago (Kergoat et al., 2014), suggesting at least 100 million years of divergence. Due to this long divergence, existing genomic resources among Coleopterans are insufficient to inform breeding efforts in T. molitor.

Here, we present a draft genome assembly for the darkling beetle T. molitor. Our work adds to a growing body of genetic resources for $T$. molitor, which currently include a mitochondrial genome (Liu and Wang, 2014), transcriptomes (Liu et al., 2015; Zhu et al., 2013), gut microbiome (Brandon et al., 2018; Jung et al., 2014), expression pattern analyses (Johnston et al., 2014; Oppert et al., 2012), and gene and peptide annotations (Liu et al., 2015; Prabhakar et al., 2007). Recently it has been demonstrated that yellow mealworms can be artificially selected for commercially desirable traits (Morales-Ramos et al., 2019). This finding highlights the need for a genome to contextualise and interpret selection outcomes, as well as to explore more efficient means of trait optimisation. Genomic sequencing and bioinformaticbased genome assembly terms mentioned in this article are explained in Supplementary material S1.

\section{Methods}

Unless specified otherwise, default settings were used for all analyses. Figures were made with RStudio (RStudio Team, 2015) using the library ggplot2 (Wickham, 2016). Raw data and final draft assembly are available in NCBI SRA database (BioProject PRJNA579236).

\section{Sequencing}

Genomic DNA (gDNA) was extracted from the head of a single adult male T. molitor Stoneville strain (MoralesRamos et al., 2019) using the Qiagen DNeasy Blood and Tissue Extraction kit (Qiagen, Germantown, MD, USA). Library preparation, sequencing, and read demultiplexing were carried out at the Indiana University (IU) School of Medicine Center for Medical Genomics Core Facility (Indianapolis, IN, USA). Quality and quantity of gDNA was first evaluated by TapeStation and Qubit with a recommendation of a molecular size range of $>50$ kilobases (kb). Then, $10 \mathrm{ng}$ of gDNA is prepared following the Chromium Genome Reagent Kit v2 User Guide, CG00043 RevB (10x Genomics, Inc., Pleasanton, CA, USA).

Along with the gel beads in emulsion (GEMs) and partitioning oil, the master mixture containing gDNA is dispensed onto a Genome Chip in separate wells, and the chip loaded to the Chromium Controller for GEM barcoding, followed by post GEM clean-up, and library preparation. The Chromium Controller prepared sequencing libraries by employing 10x Gemcode ${ }^{\mathrm{TM}}$ technology. In short, GEMs captured and tagged high-molecular-weight gDNA molecules with unique barcodes. Short reads originated from a given GEM can be computationally linked by the shared barcode to provide resolution at larger genomic scales, thereby improving assembly accuracy. In addition, since the probability of both copies at a given diploid locus receiving the same barcode is very small, the Gemcode ${ }^{\mathrm{rm}}$ technology also enables the parsing of allelic information.

The resulting library was sequenced using a custom program for $2 \times 150 \mathrm{bp}$ paired-end sequencing on Illumina NovaSeq 6000 (200 cycles, Illumina, San Diego, CA, USA). Paired-end technology allows a given genomic region to be sequenced from both complementary strands to improve assembly accuracy.

\section{De novo assembly}

Paired-end raw reads were demultiplexed with the longranger mkfastq pipeline (10x Genomics) and assembled using Supernova v2.0.0 (Weisenfeld et al., 2017) on the IU Carbonate supercomputing cluster housed at Indiana University Bloomington Data Center (Bloomington, IN, USA). The effects of coverage (assuming a genome size of $508 \mathrm{Mb}$ for $T$. molitor estimated from gametic nuclear DNA content; see Juan and Petitpierre 1991 and Gregory et al., 2007) on assembly quality were assessed by instructing Supernova to use different number of reads through the maxread parameter (Table 1). Per recommendations by 10x Genomics support, scaffolds $\geq 1000$ base pairs (bp) were retained in all assemblies. Option 'pseudohap2' was selected to output assembled haplotypes, representing haploid copies of the genome, separately. We used the program jellyfish (Marcais and Kingsford, 2011) to generate frequency distributions of unique k-mers in the unpaired raw reads after quality trimming per recommendations by 10x Genomics. Then we used GenomeScope (Vurture et al., 2017) to estimate from the k-mer distributions the genome size and repeat content.

Table 1. Values of the maxread parameter used by the Supernova assembler. The assembler uses a variety of reads that are calculated or approximated to be some coverage amount of the genome, and calculates that percentage as a function of the total number of reads generated.

$\begin{array}{lcc}\text { Coverage } & \text { Maxread } & \text { \% reads used } \\ 50 & 84,666,667 & 25.9 \\ 56 & 94,826,667 & 29 \\ 60 & 101,600,000 & 31 \\ 75 & 126,669,732 & 38.7 \\ 98 & 166,666,667 & 50.9 \\ 118 & 200,005,898 & 61.1 \\ 130 & 220,008,464 & 67.2 \\ 193 & 327,244,832 & 100\end{array}$




\section{Comparing assemblies}

Each assembly using a varying number of reads was evaluated for quality based on scaffold N50, contig N50, and number of scaffolds $\geq 1 \mathrm{~kb}$ as well as compressed scaffolds that are $\geq 10 \mathrm{~kb}$. Scaffold compression replaced continuous stretches of ambiguous bases, abbreviated Ns, by a single $\mathrm{N}$. Ambiguity is a result of low read coverage or missing data. Assembled genome size was estimated from the distribution of k-mers in reads, raw coverage was calculated by dividing the number of bases in reads prior to barcode trimming by the estimated genome size, and effective coverage was calculated by averaging base graph edges of length 10002,000 bp k-mers that appear to be diploid, and GC content was assessed. Maximum scaffold length, the number of ambiguous bases (Ns) per $100 \mathrm{~kb}$, scaffold N50, N75, L50 and L75 of uncompressed assemblies were obtained from the program Quality Assessment Tool (QUAST) v5.0.0 (Mikheenko et al., 2018). We then examined the relationship between read number and metrics used to assess genome assembly quality with regression models in RStudio, and selected the assembly with high contiguity and low ambiguity (small number of Ns) as our assembled genome for further analyses.

\section{Assembly completeness}

For the focal assembly, gene content completeness was estimated by the program Benchmarking Universal SingleCopy Orthologs (BUSCO) v3.0.2 (Simao et al., 2015; Waterhouse et al., 2018) using curated ortholog databases of Eukaryota, Arthropoda, Insecta and the insect superorder Endopterygota. Completeness is a measure of assembly quality based on the proportions of expected conserved protein-coding genes recovered from the assembly.

\section{Repetitive DNA}

RepeatMasker v4.0.7 (Smit et al., 2013) was used to screen for repetitive elements, using a Tenebrio-specific database. We also performed local BLAST searches for the 142-bp satellite motif repeats of which reportedly make up about half of the T. molitor genome (Petitpierre et al., 1988) using the CLC Genomics Workbench v. 11.0.1 (Qiagen) in which the length fraction of the read to map was 0.5 and similarity fraction of 0.8 .

\section{Gene model prediction}

Predictions of assembled sequences were done using AUGUSTUS v3.3 (Stanke and Waack, 2003) with the T. castaneum v.2012 gene database as a reference. Two analyses were performed predicting complete genes (parameter genemodel $=$ complete) and both partial and complete genes simultaneously (genemodel = partial). AUGUSTUS modelled probability distributions for different sequence types (e.g. coding DNA, mRNA, and amino acid) and gene components (e.g. exons and introns), and applied the model to the assembly to generate predictions. Partial predictions were missing certain components based on training data (e.g. partial genes $=$ missing one or more exons), therefore incomplete.

\section{Mitochondrial genome}

The mitochondrial genome (mtDNA) was isolated from the assembly using a local BLAST search in CLC Genomics Workbench (Qiagen) with the published T. molitor mitochondrial genome (accession number KF418153.1) (Liu and Wang, 2014). A consensus mtDNA sequence was extracted and annotated for the 13 mitochondrial-specific genes (Liu and Wang, 2014) to confirm its presence in our assembled genome.

\section{Results}

\section{Raw reads}

A total of $327,244,832$ reads were generated with a read quality distribution (Figure 1) wherein $90.6 \%$ of all reads had an average Q score between 29-41, which correspond to a base call accuracy between $99.9-99.99 \%$. Q scores, also known as Phred quality scores, reflect the probability that a given DNA base in a read is incorrectly determined by a sequencer. Most reads (96.99\%) did not contain ambiguities (Ns). Estimates of haploid genome size from 21-, 30-, 48-, and 74-mers ranged 221-272 $\mathrm{Mb}$ (Table 2).

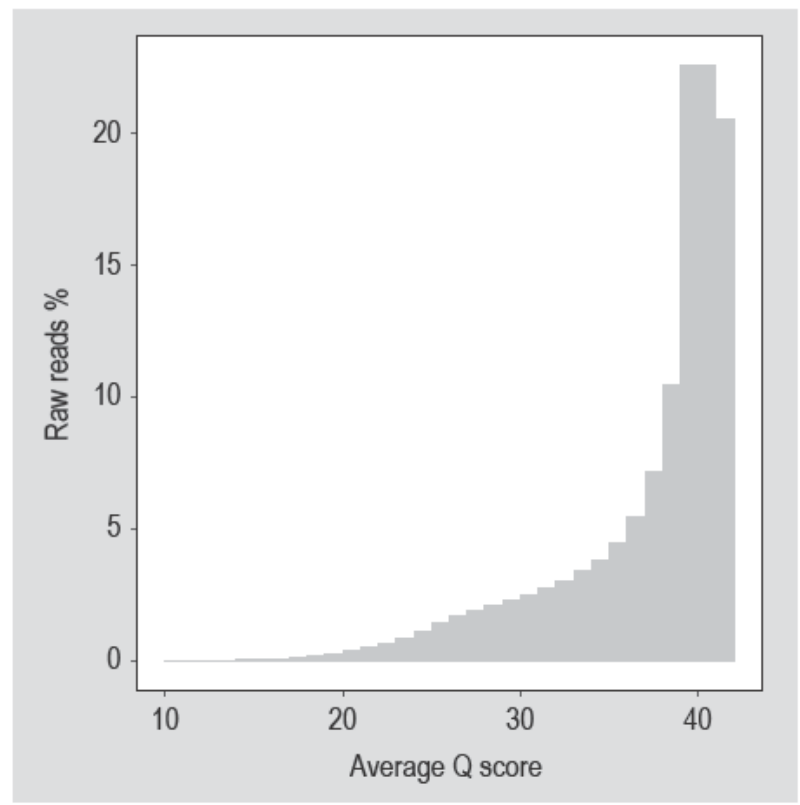

Figure 1. Sequencing quality of all raw reads expressed in $Q$ scores. Over $90 \%$ of reads scored 29 or higher on average, indicating the majority of reads are of good quality with high base-calling accuracy $(99.9-99.99 \%)$. 
Table 2. GenomeScope's estimates of haploid genome size and repeat content from raw reads based on k-mer size. Estimates were given as a range (min-max). Full genome sizes were calculated by doubling haploid estimates.

$\begin{array}{llllll}\mathbf{k} & \text { Haploid genome size }(\mathbf{M b}) & \text { Full genome size }(\mathbf{M b}) & \text { Repeat content size (Mb) } & \text { Repeat content (\%) } & \text { Model fit } \\ 21 & 244.5-245 & 489-490 & 76.7-76.8 & 31.4-31.3 & 92.07-95.03 \% \\ 30 & 221-221.9 & 442-443.8 & 40.5-40.7 & 18.3-18.3 & 94.69-96.86 \% \\ 48 & 271.7-271.9 & 543.4-543.8 & 77.1-77.1 & 28.4-28.4 & 96.85-99.06 \% \\ 74 & 261.4-262.2 & 522.8-524.4 & 58.7-58.9 & 22.5-22.5 & 97.40-99.67 \%\end{array}$

\section{Comparing assemblies}

We assessed the impact of the number of reads used for the assembly on the general outcome of the assembly. Most metrics of contiguity increased logarithmically with read number, including compressed assembly size, the number of scaffolds $\geq 10 \mathrm{~kb}$, maximum scaffold size, and scaffold N50 and N75 (Table 3; Figure 2A-D). On the other hand, the number of scaffolds of all sizes and scaffold L50 and L75 decreased exponentially with increasing numbers of reads (Table 3; Figure 2B, D). Uncompressed assembly size and number of Ns per $100 \mathrm{~kb}$ scaled linearly with read number, while estimated genome size increased exponentially (Table 3; Figure 2A, F).

Based on these results, we selected the assembly containing approximately $130 x$ coverage that scored well in contiguity metrics, with relatively high N50 and N75, low L50 and L75, the largest scaffolds, the greatest number of scaffolds $\geq 10 \mathrm{~kb}$, and low total scaffold count with relatively few Ns (Figure 2).

\section{The $130 \mathrm{x}$ assembly}

Statistics for the 130x assembly are provided in Table 4. Using CLC Genomics Workbench, we mapped all raw reads (i.e. includes reads not used in the original assembly) back to this assembly to approximate how many reads were actually used in the assembly. Overall, 306,790,136 reads, or $93.75 \%$ of total raw reads were mapped successfully (average mapping coverage across scaffolds $=238.2$ ). The assembled genome contains $36.04 \%$ GC content, within the range of known insect genomes (Archak et al., 2007). The uncompressed assembly contained $0.32 \%$ Ns estimated from the count of Ns per $100 \mathrm{~kb}$. There were 20,305 genes predicted present in the genome (includes both complete and partial genes, Figure 3, Table 5) of which $8.7-12.8 \%$ of the conserved genes were missing in our assembled genome across taxonomic databases (BUSCO, Figure 4).

Overall, RepeatMasker detected 65,915 repetitive elements accounting for $1.06 \%$ of the assembly, the majority of which were simple DNA repeats (Table 6). The repetitive landscape of T. molitor has been previously characterised based on

Table 3. Regression models of significant relationships between read number and quality metrics of eight test assemblies. Most measures of contiguity correlated positively with read numbers, and most models fit well to data, with adjusted $R^{2}$ values ranging between $0.62-0.95$.

$\begin{array}{llcccc}\text { Analysis } & \text { Regression } & \text { Coefficient } & \mathbf{t} & \boldsymbol{P} & \text { adj. } \boldsymbol{R}^{\mathbf{2}} \\ & & & & <0.001 & 0.95 \\ \text { Assembly size - uncompressed } & \text { linear } & 0.26 & 11.53 & <.501 & 0.89 \\ \text { Assembly size - compressed } & \text { logarithmic } & 115.19 & 7.58 & <0.001 & 0.99 \\ \text { Estimated genome size } & \text { exponential } & 7.06 \times 10^{-141} & 55.64 & 0.003 & 0.77 \\ \text { Total number of scaffolds } & \text { logarithmic } & -24,841 & -4.92 & <0.001 & 0.92 \\ \text { Scaffolds } \geq 10 \mathrm{~kb} & \text { logarithmic } & 2,481.8 & 8.86 & 0.012 & 0.62 \\ \text { Max scaffold size } & \text { logarithmic } & 202.95 & 3.55 & <0.001 & 0.83 \\ \text { N50 scaffold } & \text { logarithmic } & 19.31 & 5.99 & <0.001 & 0.90 \\ \text { N75 scaffold } & \text { logarithmic } & 5.07 & 7.86 & 0.007 & 0.67 \\ \text { L50 scaffold } & \text { logarithmic } & -6,383 & -3.92 & 0.003 & 0.75 \\ \text { L75 scaffold } & \text { logarithmic } & -15,622 & -4.67 & <0.001 & 0.85 \\ \text { Ns per 100 kb } & \text { linear } & 1.302 & 6.49 & \end{array}$



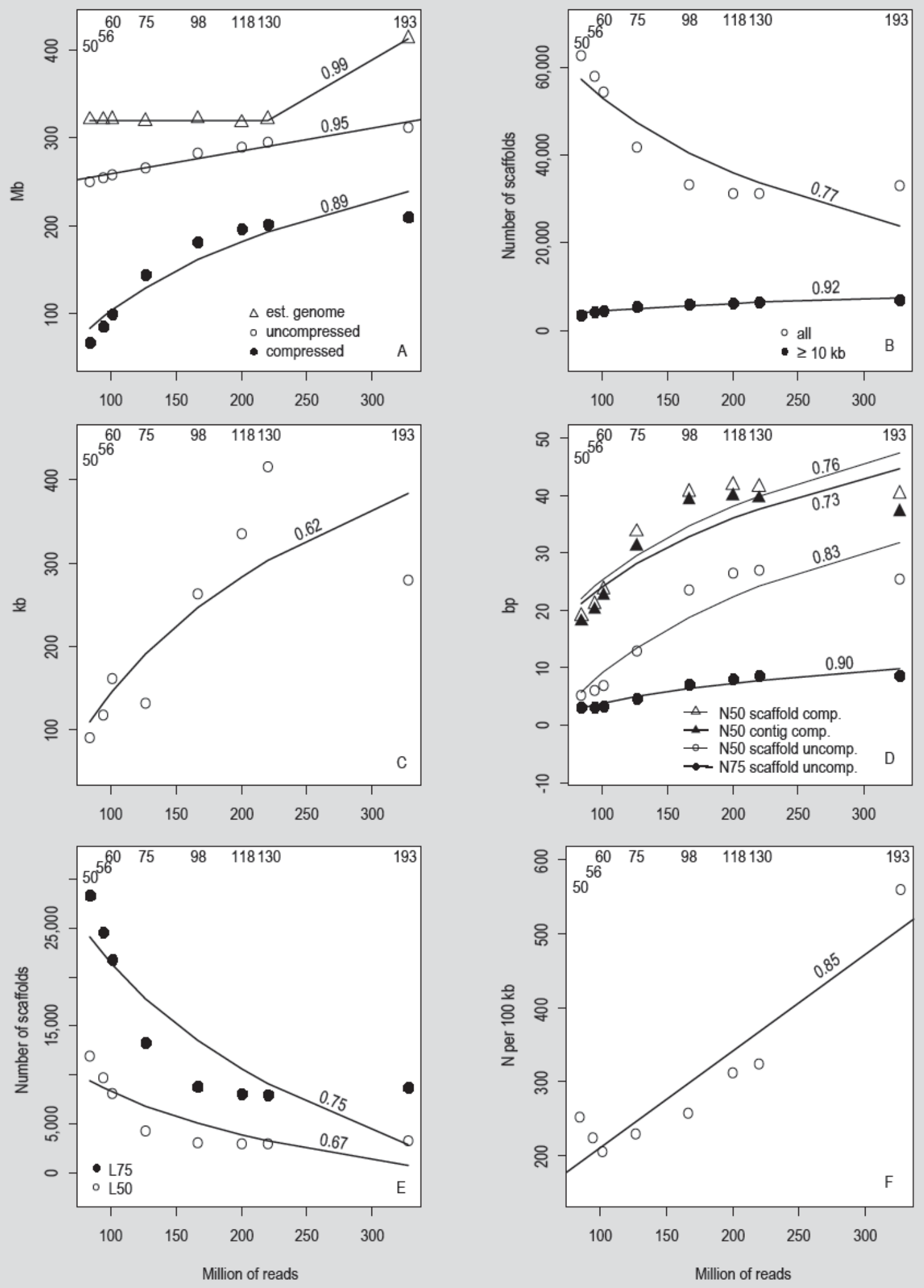

Figure 2. Effects of read number on assembly statistics. Data for eight assemblies $(50,56,60,75,98,118,130$, and 193x) were analysed with regressions. Overall, more reads resulted in larger, less fragmented assemblies at the cost of increasing ambiguity. Estimated genome size and assembly size, compressed or not, increased with the number of used reads (A). This trend was also observed in the number of scaffolds $\geq 10 \mathrm{~kb}$, but not in scaffolds of all sizes (B). Maximum scaffold size (C) and scaffold N50 and N75 (D) were also positively correlated with read number. As scaffolds increased in length, L50 and L75 (E) decreased while the amount of Ns (F) they contain increased. Associate $R^{2}$ values are printed on each regression line. 
Table 4. Assembly statistics for the $130 \mathrm{x}$ assembly. Compared to other test assemblies, the $130 \mathrm{x}$ assembly was optimal in quality, having the largest scaffold, the highest number of large scaffolds ( $\geq 10 \mathrm{~kb}$ ), high $\mathrm{N} 50$ and N75, low L50 and L75, and relatively few Ns per 100 kb. $^{1}$

\begin{tabular}{|c|c|}
\hline Assembly metrics & Value \\
\hline Uncompressed assembly size (bp) & $295,039,520$ \\
\hline Compressed assembly size (bp) & $201,953,383$ \\
\hline Estimated genome size (bp) & $321,280,000$ \\
\hline Total number of scaffolds & 31,391 \\
\hline Number of Scaffolds $\geq 10 \mathrm{~kb}$ & 6,617 \\
\hline Length of largest scaffold (bp) & $415,709^{*}$ \\
\hline Scaffold N50 (bp) & $26,962^{*} ; 41,462$ \\
\hline Scaffold N75 (bp) & $8,586^{*}$ \\
\hline Scaffold L50 & $3,050^{*}$ \\
\hline Scaffold L75 & $8,049^{*}$ \\
\hline Contig N50 (bp) & 39,478 \\
\hline Raw coverage $(x)$ & 103.403 \\
\hline Effective coverage $(\mathrm{x})$ & 59.8937 \\
\hline GC (\%) & 36.04 \\
\hline \#N's per $100 \mathrm{~kb}$ & $324.92^{*}$ \\
\hline
\end{tabular}

Table 5. Numbers of sequences predicted by AUGUSTUS for the $130 x$ assembly, including the number of partial genes predicted.

$\begin{array}{lrr}\text { Sequence type } & \text { Number of complete } & \begin{array}{l}\text { Number of genes } \\ \text { including partial genes }\end{array} \\ \text { Gene } & 16,090 & \\ \text { mRNA } & 16,090 & 20,305 \\ \text { CDS } & 16,090 & 20,305 \\ \text { Amino acid } & 16,090 & 20,305 \\ \text { Exon } & 108,412 & 20,305 \\ \text { Intron } & 92,322 & 114,393 \\ & & 103,191\end{array}$

Table 6. Types, counts, and total lengths of repetitive elements detected in the $130 \mathrm{x}$ assembly by RepeatMasker.

\begin{tabular}{lrrl} 
Sequence type & $\begin{array}{l}\text { Number of } \\
\text { elements }\end{array}$ & $\begin{array}{l}\text { Length } \\
\text { occupied (bp) }\end{array}$ & $\begin{array}{l}\text { \% of } \\
\text { sequence }\end{array}$ \\
DNA transposons & 950 & 321,487 & 0.11 \\
Unclassified & 4 & 180 & 0 \\
Small RNA & 188 & 55,767 & 0.02 \\
Satellites & 200 & 71,256 & 0.02 \\
Simple repeats & 51,723 & $2,053,642$ & 0.70 \\
Low complexity & 12,850 & 612,218 & 0.21 \\
Total & 65,915 & $3,114,550$ & 1.06 \\
\hline & \\
&
\end{tabular}

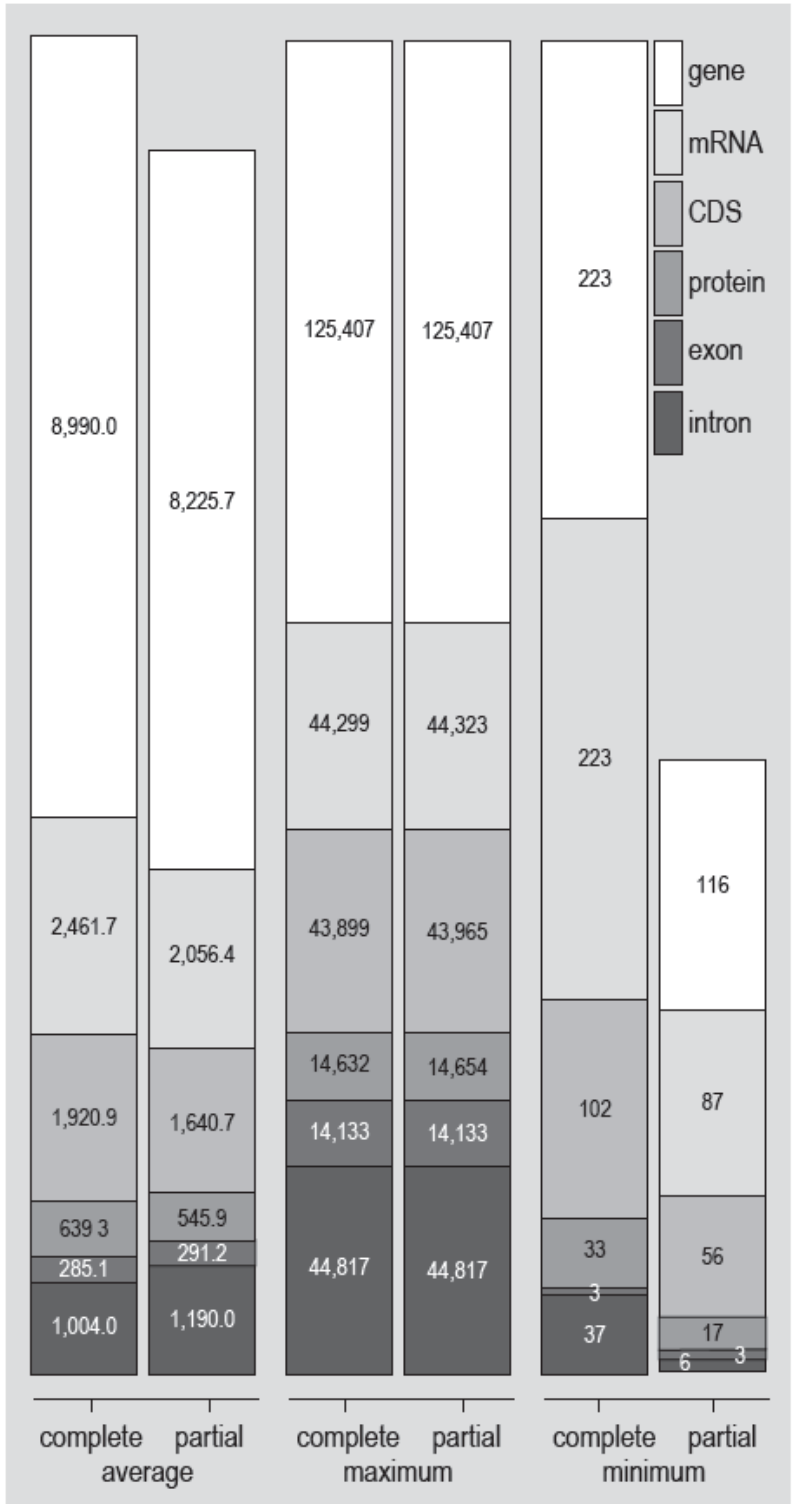

Figure 3. Size in base pairs (bp) of genes, transcripts, coding sequences, amino acid sequences, exons, and introns predicted by AUGUSTUS for the 130x assembly. AUGUSTUS was trained with annotated genes in Tribolium castaneum to model probability distributions for different types of sequences (e.g. coding DNA, mRNA, amino acid) and gene components (e.g. exons and introns), and apply the model to the assembly. 'Partial' bars included predictions that missed certain components based on the training data (e.g. partial genes $=$ missing some exons). As an example, complete genes are $8,990 \mathrm{bp}$ on average, with some as small as $223 \mathrm{bp}$ and as large as $125,407 \mathrm{bp}$. 


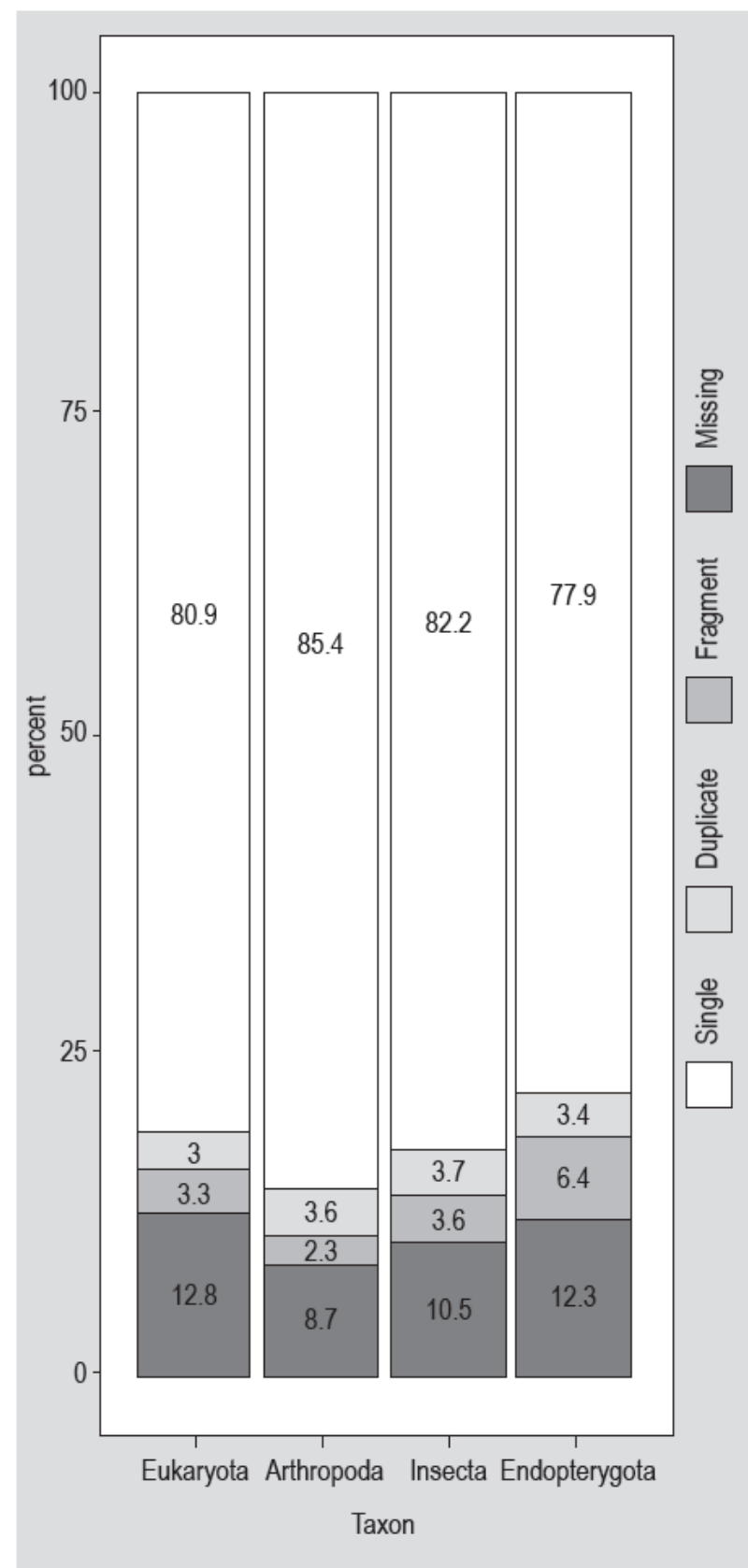

Figure 4. BUSCO results characterising gene content completeness of the $130 x$ assembly. The numbers of genes in each searched database are as follow: Eukaryota $=303$, Arthropoda $=1,066$, Insecta $=1,658$, Endopterygota $=2,442$. Counting both single-copy and duplicate genes, most Arthropoda orthologs were found ( $89 \%$ ) while Endopterygota had the fewest hits $(81.3 \%)$. the abundant presence of the 142-bp satellite sequence (Petitpierre et al., 1988). RepeatMasker detected 334 instances of this satellite across 155 scaffolds, accounting for $0.02 \%$ of base pairs in the uncompressed assembly. Similarly in a BLAST analysis, the simple repeat was detected in 112 scaffolds (BLASTn overlap $>90 \%$, identity $\geq 69 \%$; average mapping coverage $=58,020.2$ ), with repeating unit ranged from one to 25 times (average $=4.11 \pm 5 \mathrm{SD}$ ) and accounted for roughly $0.015 \%$ of the uncompressed assembly. In the scaffold with 25 repeats, the satellite sequence measured 3,350 bp or $35.7 \%$ of the scaffold length. When raw reads were mapped to the satellite sequence, $24.75 \%$ of the reads mapped, a substantial portion of reads from the genome that are associated with this repetitive unit.

The mitochondrial genome was assembled in a single scaffold with an average coverage of 18,051 and a length of $15,785 \mathrm{bp}(72.4 \% \mathrm{~A} / \mathrm{T}, 0 \% \mathrm{~N})$ and $99.75 \%$ sequence similarity to the published mtDNA genome, containing eight gaps and 31 nucleotide variants. Amino acid sequences of the 13 mitochondrial protein-coding genes were used as query against the consensus sequence translated across all reading frames. Significant BLAST hits with E-values $=0$ and without internal stop codons suggested the recovered scaffold was indeed the mtDNA genome. For three genes in our mitochondrial assembly, amino-acid polymorphisms were detected relative to the queries: cytochrome b (leucine $\rightarrow$ methionine at position 305), cytochrome c oxidase subunit III (threonine $\rightarrow$ alanine at position 173), and NADH dehydrogenase subunit 4 (valine $\rightarrow$ methionine at position 379 ). The mtDNA can be accessed via NCBI (accession number MT041765).

\section{Discussion}

We present the first linked-read assembled draft genome for the economically important insect species $T$. molitor. Several animal genomes have been assembled with this sequencing technology (Jones et al., 2017; Ozerov et al., 2018; Wang et al., 2019), which has the advantages of producing less fragmented assemblies than standard short reads (Huddleston et al., 2014; Pareek et al., 2011), while avoiding the high rate of sequencing error typically associated with long-read technologies (Del Angel et al., 2018; English et al., 2012). As such, our genome is a more complete resource for the community when working to optimise insects as food and feed.

Assuming a complete genome with $508 \mathrm{Mb}$ (Gregory et al., 2007; Juan and Petitpierre, 1991), the Supernova assembler's estimates of genome size using the distribution of $\mathrm{k}$-mers in raw reads suggested that sequencing only captured roughly $62-81 \%$ of the total content (Figure $2 \mathrm{~A}$ ). While this may well be the case, several factors can produce genome assemblies that are smaller than the expected sizes. For example, the repetitive landscape may obscure 
the real genome size. If the 142 -bp simple repeat is actually present in $\sim 25 \%$ of the reads, then the assembly would be smaller due to reads mapping to that specific region. Based on uncompressed and compressed sizes, our 130x draft assembly captured $58 \%$ of the structure and $40 \%$ of the genetic information of the genome (Table 4). The missing portion is likely attributable in part to the well-characterised 142-bp satellite which is estimated to make up between 44 and $56 \%$ of the genome (Petitpierre et al., 1988). In the final assembly, however, we were only able to capture a small part of this repetitive element which accounted for about $0.02 \%$ of the assembly size. Indeed, the successful assembly of scaffolds containing repetitive elements demonstrated the advantage of linked-reads in piecing together difficult-toalign regions. Estimated genome size was stable around 310 $\mathrm{Mb}$ when $26-67 \%$ of reads were utilised by the assembler, and increased to $413 \mathrm{Mb}$ when all reads were used (Table 1, Figure 2A). On the other hand, independent estimates of genome size by GenomeScope computed from a range of $\mathrm{k}$-mers suggested that most or all of the genome was fully sequenced including repetitive content (Table 2).

A drawback of using more reads during assembly is that more ambiguous bases tend to be introduced, and we saw greater value in assembling a genome that maximises the amount of genic information. Our selection of the 130x assembly was based on numerous statistics that suggest it was the most optimal in balancing usable information and noise. Despite missing a portion of the genome, our assembly performed well on the gene content completeness benchmark with $>87 \%$ of conserved orthologs (complete or partial) detected for each taxonomic level (Figure 4). In addition, gene model prediction suggested the current assembly contains $16,090-20,305$ coding loci, similar to figures found in T. castaneum (Richards et al., 2008) and other beetles (McKenna, 2018).

Variation at the amino acid level observed between our mitochondrial sequence and the published mitochondrial genome can probably be explained in part by the history of the specimens. Ours came from a strain kept for over a decade at the National Biological Control Laboratory in Stoneville, MS, USA with ancestry in the domestic pet-food industry (Morales-Ramos et al., 2019), while the published mtDNA was derived from an inbred laboratory line established in 2008 in China (Liu and Wang, 2014). These two populations likely have undergone multiple genetic bottlenecks, and have been reared in different environments with variable growing conditions and stressors. In fact, the Stoneville strain experienced a period of population contraction during a selection experiment for larger body size, which has been in effect for at least six to seven generations prior to sequencing (Morales-Ramos et al., 2019). These factors may be sufficient to drive divergence and lead to amino acid substitutions.
The trade-offs between beneficial and undesirable traits have previously been a major obstacle to efficient mass rearing efforts of insects. For example, tephretid fruit flies, when mass-reared, show increased fecundity and faster developmental times, but experience trade-offs such as lower resilience to stress, and reduced longevity (Gilchrist et al., 2012). Selection for larger body size in the yellow mealworm is correlated with a trade-off in early larval mortality (Morales-Ramos et al., 2019). It is unknown how these traits are linked, and what the genetic basis might be for such trade-offs. With genomic tools, researchers can better understand the genetic basis of phenotypes and test hypotheses of trait linkages to resolve commercially costly trade-offs.

We present a high-quality draft genome of the yellow mealworm T. molitor, which is now available to researchers and industry alike, to further advance our understanding of mealworm biology. Ongoing efforts to optimise mass-rearing of the yellow mealworm are expected to be significantly facilitated by this draft genome. With this resource, we contribute to the study of genomics and evolution in beetles, but we also aim to elevate the yellow mealworm as a competitive protein source for food production and as an animal feed ingredient.

\section{Acknowledgements}

This work was supported with financial support from the School of Science at Indiana University Purdue University Indianapolis (IUPUI), and the Department of Biology at IUPUI. The authors would like to thank the Core Genomics Facility (specifically Xiaoling Xuei and Yunlong Liu) for help regarding the sequencing of the specimen. Computing support was in the form of use of Indiana University from the National Center for Genome Analysis at Indiana University is supported by the National Science Foundation (DBI-1062432, ABI-1458641, ABI1759906). The work used Indiana University's Carbonate Cluster, funded by the Lilly Endowment Inc., through its support for the Indiana University Pervasive Technology Institute, and by the National Science Foundation under Grant No.CNS-0521433. This material is based upon work supported by the Defense Advanced Research Projects Agency (DARPA) under Contract No. D17PC00196. Any opinions, findings and conclusions or recommendations expressed in this material are those of the author(s) and do not necessarily reflect the views of the Defense Advanced Research Projects Agency (DARPA); or its Contracting Agent, the U.S. Department of the Interior, Interior Business Center, Acquisition Services Directorate, Division III.

\section{Conflict of interest}

The authors declare no competing interests. 


\section{Supplementary material}

Supplementary material can be found online at https://doi. org/10.3920/JIFF2019.0057.

Supplementary material S1. Glossary of genomic sequencing and bioinformatic-based genome assembly terms.

\section{References}

Archak, S., Meduri, E., Kumar, P.S. and Nagaraju, J., 2007. InSatDb: a microsatellite database of fully sequenced insect genomes. Nucleic Acids Research 35: D36-D39. https://doi.org/10.1093/nar/gkl778

Barton, P.S., Gibb, H., Manning, A.D., Lindenmayer, D.B. and Cunningham, S.A., 2011. Morphological traits as predictors of diet and microhabitat use in a diverse beetle assemblage. Biological Journal of the Linnean Society 102: 301-310. https://doi.org/10.1111/ j.1095-8312.2010.01580.x

Beutel, R.G. and Leschen, R.A.B., 2014. Handbook of zoology, Coleoptera. Vol. 3. Morphology and systematics (Phytophaga). Walter de Gruyter, Berlin, Germany.

Brandon, A.M., Gao, S.H., Tian, R.M., Ning, D.L., Yang, S.S., Zhou, J.Z., Wu, W.M. and Criddle, C.S., 2018. Biodegradation of polyethylene and plastic mixtures in mealworms (larvae of Tenebrio molitor) and effects on the gut microbiome. Environmental Science \& Technology 52: 6526-6533. https://doi.org/10.1021/acs.est.8b02301

Choi, I.-H., Kim, J.-M., Kim, N.-J., Kim, J.-D., Park, C., Park, J.-H. and Chung, T.-H., 2018. Replacing fish meal by mealworm (Tenebrio molitor) on the growth performance and immunologic responses of white shrimp (Litopenaeus vannamei). Acta Scientiarum 40: e39077. https://doi.org/10.4025/actascianimsci.v40i1.39077

Del Angel, V.D., Hjerd, E., Sterck, L. and 2018. Ten steps to get started in genome assembly and annotation. F1000Research 148. https:// doi.org/10.12688/f1000research.13598.1

English, A.C., Richards, S., Han, Y., Wang, M., Vee, V., Qu, J.X., Qin, X., Muzny, D.M., Reid, J.G., Worley, K.C. and Gibbs, R.A., 2012. Mind the gap: upgrading genomes with pacific biosciences RS long-read sequencing technology. PLoS ONE 7: e47768. https:// doi.org/10.1371/journal.pone.0047768

Food and Agriculture Organisation (FAO), 2017. Insects for food and feed. FAO, Rome, Italy. Available at: http://www.fao.org/edibleinsects/en/

Gilchrist, A.S., Cameron, E.C., Sved, J.A. and Meats, A.W., 2012. Genetic consequences of domestication and mass rearing of pest fruit fly Bactrocera tryoni (Diptera: Tephritidae). Journal of Economic Entomology 105: 1051-1056. https://doi.org/10.1603/ Ec11421

Gregory, T.R., Nicol, J.A., Tamm, H., Kullman, B., Kullman, K., Leitch, I.J., Murray, B.G., Kapraun, D.F., Greilhuber, J. and Bennett, M.D., 2007. Eukaryotic genome size databases. Nucleic Acids Research 35: D332-D338. https://doi.org/10.1093/nar/gkl828
Henry, M.A., Gasco, L., Chatzifotis, S. and Piccolo, G., 2018. Does dietary insect meal affect the fish immune system? The case of mealworm, Tenebrio molitor on European sea bass, Dicentrarchus labrax. Developmental and Comparative Immunology 81: 204-209. https://doi.org/10.1016/j.dci.2017.12.002

Huddleston, J., Ranade, S., Malig, M., Antonacci, F., Chaisson, M., Hon, L., Sudmant, P.H., Graves, T.A., Alkan, C., Dennis, M.Y., Wilson, R.K., Turner, S.W., Korlach, J. and Eichler, E.E., 2014. Reconstructing complex regions of genomes using long-read sequencing technology. Genome Research 24: 688-696. https:// doi.org/10.1101/gr.168450.113

Johnston, P.R., Makarova, O. and Rolff, J., 2014. Inducible defenses stay up late: temporal patterns of immune gene expression in Tenebrio molitor. G3-Genes Genomes Genetics 4: 947-955. https://doi. org/10.1534/g3.113.008516

Jones, S.J., Haulena, M., Taylor, G.A., Chan, S., Bilobram, S., Warren, R.L., Hammond, S.A., Mungall, K.L., Choo, C., Kirk, H., Pandoh, P., Ally, A., Dhalla, N., Tam, A.K.Y., Troussard, A., Paulino, D., Coope, R.J.N., Mungall, A.J., Moore, R., Zhao, Y.J., Birol, I., Ma, Y., Marra, M. and Jones, S.J.M., 2017. The genome of the Northern Sea otter (Enhydra lutris kenyoni). Genes 8: 379. https://doi.org/10.3390/genes8120379

Juan, C. and Petitpierre, E., 1991. Evolution of genome size in darkling beetles (Tenebrionidae, Coleoptera). Genome 34: 169-173. https:// doi.org/10.1139/g91-026

Jung, J., Heo, A., Park, Y.W., Kim, Y.J., Koh, H. and Park, W., 2014. Gut microbiota of Tenebrio molitor and their response to environmental change. Journal of Microbiology and Biotechnology 24: 888-897. https://doi.org/10.4014/jmb.1405.05016

Kergoat, G.J., Bouchard, P., Clamens, A.L., Abbate, J.L., Jourdan, H., Jabbour-Zahab, R., Genson, G., Soldati, L. and Condamine, F.L., 2014. Cretaceous environmental changes led to high extinction rates in a hyperdiverse beetle family. BMC Evolutionary Biology 14: 220. https://doi.org/10.1186/s12862-014-0220-1

Liu, L.N. and Wang, C.Y., 2014. Complete mitochondrial genome of yellow meal worm(Tenebrio molitor). Zoological Research 35: 537-545.

Liu, S., Shi, X.X., Jiang, Y.D., Zhu, Z.J., Qian, P., Zhang, M.J., Yu, H., Zhu, Q.Z., Gong, Z.J. and Zhu, Z.R., 2015. De novo analysis of the Tenebrio molitor (Coleoptera: Tenebrionidae) transcriptome and identification of putative glutathione S-transferase genes. Applied Entomology and Zoology 50: 63-71. https://doi.org/10.1007/s13355014-0305-8

Marcais, G. and Kingsford, C., 2011. A fast, lock-free approach for efficient parallel counting of occurrences of k-mers. Bioinformatics 27: 764-770. https://doi.org/10.1093/bioinformatics/btr011

McKenna, D.D., 2018. Beetle genomes in the $21^{\text {st }}$ century: prospects, progress and priorities. Current Opinion in Insect Science 25: 7682. https://doi.org/10.1016/j.cois.2017.12.002

Mckenna, D.D., Wild, A.L., Kanda, K., Bellamy, C.L., Beutel, R.G., Caterino, M.S., Farnum, C.W., Hawks, D.C., Ivie, M.A., Jameson, M.L., Leschen, R.A.B., Marvaldi, A.E., Mchugh, J.V., Newton, A.F., Robertson, J.A., Thayer, M.K., Whiting, M.F., Lawrence, J.F., Slipinski, A., Maddison, D.R. and Farrell, B.D., 2015. The beetle tree of life reveals that Coleoptera survived end-Permian mass extinction to diversify during the Cretaceous terrestrial revolution. Systematic Entomology 40: 835-880. https://doi.org/10.1111/syen.12132 
Mikheenko, A., Prjibelski, A., Saveliev, V., Antipov, D. and Gurevich, A., 2018. Versatile genome assembly evaluation with QUAST-LG. Bioinformatics 34: 142-150. https://doi.org/10.1093/bioinformatics/ bty 266

Morales-Ramos, J.A., Kelstrup, H.C., Rojas, M.G. and Emery, V., 2019. Body mass increase induced by eight years of artificial selection in the yellow mealworm (Coleoptera: Tenebrionidae) and life history trade-offs. Journal of Insect Science 19: 4. https://doi.org/10.1093/ jisesa/iey110

Oonincx, D.G.A.B. and De Boer, I.J.M., 2012. Environmental impact of the production of mealworms as a protein source for humans - a life cycle assessment. PLoS ONE 7: e51145. https://doi.org/10.1371/ journal.pone. 0051145

Oppert, B., Dowd, S.E., Bouffard, P., Li, L., Conesa, A., Lorenzen, M.D., Toutges, M., Marshall, J., Huestis, D.L., Fabrick, J., Oppert, C. and Jurat-Fuentes, J.L., 2012. Transcriptome profiling of the intoxication response of Tenebrio molitor larvae to Bacillus thuringiensis Cry3Aa protoxin. PLoS ONE 7: e34624. https://doi.org/10.1371/journal. pone.0034624

Ozerov, M.Y., Ahmad, F., Gross, R., Pukk, L., Kahar, S., Kisand, V. and Vasemagi, A., 2018. Highly continuous genome assembly of Eurasian perch (Perca fluviatilis) using linked-read sequencing. G3-Genes Genomes Genetics 8: 3737-3743. https://doi.org/10.1534/ g3.118.200768

Pareek, C.S., Smoczynski, R. and Tretyn, A., 2011. Sequencing technologies and genome sequencing. Journal of Applied Genetics 52: 413-435. https://doi.org/10.1007/s13353-011-0057-x

Petitpierre, E., Gatewood, J.M. and Schmid, C.W., 1988. Satellite DNA from the beetle Tenebrio-Molitor. Experientia 44: 498-499. https:// doi.org/10.1007/Bf01958925

Poveda, J., Jimenez-Gomez, A., Saati-Santamaria, Z., UsateguiMartin, R., Rivas, R. and Garcia-Fraile, P., 2019. Mealworm frass as a potential biofertilizer and abiotic stress tolerance-inductor in plants. Applied Soil Ecology 142: 110-122. https://doi.org/10.1016/j. apsoil.2019.04.016

Prabhakar, S., Chen, M.S., Elpidina, E.N., Vinokurov, K.S., Smith, C.M., Marshall, J. and Oppert, B., 2007. Sequence analysis and molecular characterization of larval midgut cDNA transcripts encoding peptidases from the yellow mealworm, Tenebrio molitor L. Insect Molecular Biology 16: 455-468. https://doi.org/10.1111/ j.1365-2583.2007.00740.x

Richards, S., Gibbs, R.A., Weinstock, G.M., Brown, S.J., Denell, R., Beeman, R.W., Gibbs, R., Bucher, G., Friedrich, M., Grimmelikhuijzen, C.J.P., Klingler, M., Lorenzen, M.D., Roth, S., Schroder, R., Tautz, D., Zdobnov, E.M., Muzny, D., Attaway, T., Bell, S., Buhay, C.J., Chandrabose, M.N., Chavez, D., Clerk-Blankenburg, K.P., Cree, A., Dao, M., Davis, C., Chacko, J., Dinh, H., DuganRocha, S., Fowler, G., Garner, T.T., Garnes, J., Gnirke, A., Hawes, A., Hernandez, J., Hines, S., Holder, M., Hume, J., Jhangiani, S.N., Joshi, V., Khan, Z.M., Jackson, L., Kovar, C., Kowis, A., Lee, S., Lewis, L.R., Margolis, J., Morgan, M., Nazareth, L.V., Nguyen, N., Okwuonu, G., Parker, D., Ruiz, S.J., Santibanez, J., Savard, J., Scherer, S.E., Schneider, B., Sodergren, E., Vattahil, S., Villasana, D., White, C.S., Wright, R., Park, Y., Lord, J., Oppert, B., Brown, S., Wang, L.J., Savard, J., Liu, Y., Worley, K., Elsik, C.G., Reese, J.T., Elhaik, E., Landan, G., Graur, D., Arensburger, P., Atkinson, P., Beidler,
J., Demuth, J.P., Drury, D.W., Du, Y.Z., Fujiwara, H., Maselli, V., Osanai, M., Robertson, H.M., Tu, Z., Wang, J.J., Wang, S.Z., Song, H., Zhang, L., Sodergren, E., Werner, D., Stanke, M., Morgenstern, B., Solovyev, V., Kosarev, P., Brown, G., Chen, H.C., Ermolaeva, O., Hlavina, W., Kapustin, Y., Kiryutin, B., Kitts, P., Maglott, D., Pruitt, K., Sapojnikov, V., Souvorov, A., Mackey, A.J., Waterhouse, R.M., Wyder, S., Zdobnov, E.M., Kriventseva, E.V., Kadowaki, T., Bork, P., Aranda, M., Bao, R.Y., Beermann, A., Berns, N., Bolognesi, R., Bonneton, F., Bopp, D., Butts, T., Chaumot, A., Denell, R.E., Ferrier, D.E.K., Gordon, C.M., Jindra, M., Klingler, M., Lan, Q., Lattorff, H.M.G., Laudet, V., Von Levetsow, C., Liu, Z.Y., Lutz, R., Lynch, J.A., Da Fonseca, R.N., Posnien, N., Reuter, R., Roth, S., Schinko, J.B., Schmitt, C., Schoppmeier, M., Shippy, T.D., Simonnet, F., MarquesSouza, H., Tomoyasu, Y., Trauner, J., Van der Zee, M., Vervoort, M., Wittkopp, N., Wimmer, E.A., Yang, X.Y., Jones, A.K., Sattelle, D.B., Ebert, P.R., Nelson, D., Scott, J.G., Muthukrishnan, S., Kramer, K.J., Arakane, Y., Zhu, Q.S., Hogenkamp, D., Dixit, R., Jiang, H.B., Zou, Z., Marshall, J., Elpidina, E., Vinokurov, K., Oppert, C., Evans, J., Lu, Z.Q., Zhao, P.C., Sumathipala, N., Altincicek, B., Vilcinskas, A., Williams, M., Hultmark, D., Hetru, C., Hauser, F., Cazzamali, G., Williamson, M., Li, B., Tanaka, Y., Predel, R., Neupert, S., Schachtner, J., Verleyen, P., Raible, F., Walden, K.K.O., Robertson, H.M., Angeli, S., Foret, S., Schuetz, S., Maleszka, R., Miller, S.C., Grossmann, D. and Tribolium Genome Sequencing Consortium, 2008. The genome of the model beetle and pest Tribolium castaneum. Nature 452: 949-955. https://doi.org/10.1038/nature06784.

Simao, F.A., Waterhouse, R.M., Ioannidis, P., Kriventseva, E.V. and Zdobnov, E.M., 2015. BUSCO: assessing genome assembly and annotation completeness with single-copy orthologs. Bioinformatics 31: 3210-3212. https://doi.org/10.1093/bioinformatics/btv351

Smit, A.F.A., Hubley, R. and Green, P., 2013. RepeatMasker Open-4.0. Available at: http://www.repeatmasker.org

Stanke, M. and Waack, S., 2003. Gene prediction with a hidden Markov model and a new intron submodel. Bioinformatics 19: Ii215-Ii225. https://doi.org/10.1093/bioinformatics/btg1080

Van Broekhoven, S., Mota Gutierrez, J., De Rijk, T.C., De Nijs, W.C.M. and Van Loon, J.J.A., 2017. Degradation and excretion of the Fusarium toxin deoxynivalenol by an edible insect, the Yellow mealworm (Tenebrio molitor L.). World Mycotoxin Journal 10: 169-169.

Veldkamp, T. and Bosch, G., 2015. Insects: a protein-rich feed ingredient in pig and poultry diets Animal Frontiers 5: 45-50.

Vurture, G.W., Sedlazeck, F.J., Nattestad, M., Underwood, C.J., Fang, H., Gurtowski, J. and Schatz, M.C., 2017. GenomeScope: fast referencefree genome profiling from short reads. Bioinformatics 33: 22022204. https://doi.org/10.1093/bioinformatics/btx153

Wang, W., Yan, H.J., Chen, S.Y., Li, Z.Z., Yi, J., Niu, L.L., Deng, J.P., Chen, W.G., Pu, Y., Jia, X.B., Qu, Y., Chen, A., Zhong, Y., Yu, X.M., Pang, S., Huang, W.L., Han, Y., Liu, G.J. and Yu, J.Q., 2019. The sequence and de novo assembly of hog deer genome. Scientific Data 6: 180305. https://doi.org/10.1038/sdata.2018.305

Waterhouse, R.M., Seppey, M., Simao, F.A., Manni, M., Ioannidis, P., Klioutchnikov, G., Kriventseva, E.V. and Zdobnov, E.M., 2018. BUSCO applications from quality assessments to gene prediction and phylogenomics. Molecular Biology and Evolution 35: 543-548. https://doi.org/10.1093/molbev/msx319 
Weisenfeld, N.I., Kumar, V., Shah, P., Church, D.M. and Jaffe, D.B., 2017. Direct determination of diploid genome sequences. Genome Research 27: 757-767. https://doi.org/10.1101/gr.214874.116

Wickham, H., 2016. ggplot2: elegant graphics for data analysis. Springer International Publishing, New York, NY, USA.

Zhang, Z.Q., Hooper, J.N.A., Van Soest, R.W.M., Pisera, A., Crowther, A.L., Tyler, S., Schilling, S., Eschmeyer, W.N., Fong, J.D., Blackburn, D.C., Wake, D.B., Wilson, D.E., Reeder, D.M., Fritz, U., Hodda, M., Guidetti, R., Bertolani, R., Mayer, G., Oliveira, I.D., Zhang, Z.Q., Adrain, J.M., Bamber, R.N., Kury, A.B., Prendini, L., Harvey, M.S., Beaulieu, F., Dowling, A.P.G., Klompen, H., De Moraes, G.J., Walter, D.E., Zhang, Z.Q., Fan, Q.H., Pesic, V., Smit, H., Bochkov, A.V., Khaustov, A.A., Baker, A., Wohltmann, A., Wen, T.H., Amrine, J.W., Beron, P., Lin, J.Z., Gabrys, G., Husband, R., Bolton, S., Uusitalo, M., Zhang, Z.Q., Schatz, H., Behan-Pelletier, V.M., OConnor, B.M., Norton, R.A., Dunlop, J.A., Penney, D., Minelli, A., Shear, W., Ahyong, S.T., Lowry, J.K., Alonso, M., Bamber, R.N., Boxshall, G.A., Castro, P., Gerken, S., Karaman, G.S., Goy, J.W., Jones, D.S., Meland, K., Rogers, D.C., Svavarsson, J., Janssens, F., Christiansen, K.A., Ingrisch, S., Brock, P.D., Marshall, J., Beccaloni, G.W., Eggleton,
P., Mound, L.A., Slipinski, S.A., Leschen, R.A.B., Lawrence, J.F., Holzenthal, R.W., Morse, J.C., Kjer, K.M., Van Nieukerken, E.J., Kaila, L., Kitching, I.J., Kristensen, N.P., Lees, D.C., Minet, J., Mitter, C., Mutanen, M., Regier, J.C., Simonsen, T.J., Wahlberg, N., Yen, S.H., Zahiri, R., Adamski, D., Baixeras, J., Bartsch, D., Bengtsson, B.A., Brown, J.W., Bucheli, S.R., Davis, D.R., De Prins, J., De Prins, W., Epstein, M.E., Gentili-Poole, P., Gielis, C., Hattenschwiler, P., Hausmann, A., Holloway, J.D., Kallies, A., Karsholt, O., Kawahara, A.Y., Koster, S., Kozlov, M.V., Lafontaine, J.D., Lamas, G., Landry, J.F., Lee, S., Nuss, M., Park, K.T., Penz, C., Rota, J., Schintlmeister, A., Schmidt, B.C., Sohn, J.C., Solis, M.A., Tarmann, G.M., Warren, A.D., Weller, S., Yakovlev, R.V., Zolotuhin, V.V., Zwick, A., Pape, T., Blagoderov, V., Mostovski, M.B., Emig, C.C., Segers, H., Monks, S. and Richardson, D.J., 2011. Animal biodiversity: an outline of higher-level classification and taxonomic richness. Zootaxa: 7-237. https://doi.org/10.11646/zootaxa.3148.1.3

Zhu, J.Y., Yang, P., Zhang, Z., Wu, G.X. and Yang, B., 2013. Transcriptomic immune response of Tenebrio molitor pupae to parasitization by Scleroderma guani. PLoS ONE 8: e54411. https:// doi.org/10.1371/journal.pone.0054411 
